\title{
Research article \\ A study to compare task-based mirror therapy versus constraint induced movement therapy for hand function in hemiplegic subjects
}

\author{
Preetha K. ${ }^{1}$, Vimala U. ${ }^{1}$, Kamalakannan M. ${ }^{2}$ \\ ${ }^{1}$ Undergraduate Student, ${ }^{2}$ Assistant Professor, Saveetha College of Physiotherapy, SIMATS, Chennai, Tamil Nadu, India
}

(Received: February $2021 \quad$ Revised: July $2021 \quad$ Accepted: August 2021)

Corresponding author: Kamalakannan M. Email: kamal1712@gmail.com

\begin{abstract}
Introduction and Aim: To find the effectiveness of task-based mirror therapy when compared with constraintinduced movement therapy for hand function in hemiplegic subjects. Stroke is a major health problem in the world. According to various researches, $70 \%-80 \%$ of the patients have the upper extremity impairment then lower extremity. Task-based mirror therapy can be an effective management for premotor cortex impairment. Constraintinduced movement therapy forms the new neural pathway when used properly.
\end{abstract}

Materials and Methods: 30 patients with subacute CVA (cerebrovascular attack) were enrolled and randomly divided into two groups: constraint-induced movement therapy (CIMT) and task-based mirror therapy (TMBT). The full-Meyer motor function assessment was evaluated 4 weeks after treatment.

Results: The results showed that mean value of TMBT (pre-test -5.733 , post-test- 9.86) and SD of (pre-test- 1.67, post-test- 1.92) and in CIMT (pre-test-6.3, post-test-11.13) and SD (Pre-test-1.45, Post-test -1.36).

Conclusion: This study shows that CIMT group showed more improvement than TMBT group.

Keywords: Hemiplegia; constraint-induced movement therapy; task-based mirror therapy; stroke; acute stroke.

\section{INTRODUCTION}

$\mathrm{S}$ troke is a worldwide major health problem. It was the second most common cause of death worldwide and the third most common cause of disability. The significant increase in stroke burden worldwide. Over the last two and a half decades (1). Approximately 20 million people each year will suffer from stroke and those 5 million will not survive (2). $70 \%-80 \%$ of patient who survives from stroke have been reported to have persistent impairment of the upper limb movement. "Hemiplegia is a paralysis of one side of the body due to pyramidal tract lesion at any point from its origin in the cerebral cortex down to the fifth cervical segment. Distal parts recovery is poor in stroke patients especially hand and foot. In most of the stroke patients Up to $85 \%$ of them show an initial deficit in the hand function only. Three to six months later, problems remain in 55\%-75\% of patient (3-5). While recovery of hand function is poor in a significant number of patients, leg function has proven to be less of a problem.

Mirror therapy is a useful intervention for the rehabilitation of upper limb functions following stroke. The mirror is an inexpensive and patientcentered method. This method focuses on healthy and unaffected limbs and makes the patients feel they still have intact limbs after having them paralyzed (6-8). Mirror therapy is responsible for transferring the visual inputs resulting from the unaffected limb movement into the limb. Furthermore, the mirror neuron can counterbalance different visual inputs, proprioceptive inputs, motor commands. They are evidence showing that mirror has a quick effect on restarting the motor units and consequently increase the reorganization and neuroplasticity or premotor cortex. Moreover, improvement in upper limb function and increased self-care ability or also been reported in sub-acute patients treating with mirror therapy (9).

Constraint-induced movement therapy technique produce the use of the affected arm by limiting the use of the unaffected side with a sling or splint. This is denoting the term cortical reorganization or neuroplasticity. The main difference between MT and CIMT is the part of the extremity engaged in the rehabilitation exercise. In mirror therapy, the unaffected arm is moral, and the patient receives only visual feedback from the mirror. However, in CIMT, the unaffected arm is voluntarily engaged and hence both types facilitate motor function recovery.

\section{MATERIALS AND METHODS}

The material used: Certain tasks are done with pebbles, smiley balls, coin, pegboard. Inclusion criteria: The patient was supposed to score higher than 21 in the Minimental scale examination test (MMSE). Recovery stage of stroke. Mild to Moderate motor impairment, level 3 to 5 in the functional test for the hemiplegic upper extremity. Middle cerebral artery affected subjects. Mild to moderate degree of spasticity in all joints of the affected subjects. The patient could perform an active extension of the affected wrist at an angle $>10^{\circ}$ and active abduction of the affected thumb at an angle of $>10^{\circ}$. Exclusion 
criteria: History of other neurological, orthopedic disorders that affect upper limb function. Serious unilateral neglect star cancellation test $\leq 44-154$.

\section{Procedure}

A total of 30 subjects were selected using convenience sampling technique based on inclusion and exclusion criteria. The study was explained to the patient and written consent will be obtained from each subject. Subjects were be allocated into two groups. Group A with 15 subjects was treated with task-based mirror therapy and Group B with 15 subjects was treated with constraint-induced movement therapy.

\section{Constraint-Induced Movement Therapy (CIMT)}

The patient is asked to sit on the chair with back support. A patient's unaffected hand is tied with a triangular bandage or splint and the patient asks to perform the exercise through the affected hand. CIMT is a multifaceted intervention designed to promote increased use of affected upper extremity, for up to 6 hours a day for 4 days week $/ 4$ weeks and the patient nonparetic arm restrained from the use by having the patient placing a mitt on the hand or a sling on the unaffected hand, forcing the use of the affected limb to promote purposeful movements when performing functional tasks.

\section{Task Based Mirror Therapy}

The patient is asked to sit and facing towards a table on a chair with a backrest with legs placing on the ground. The mirror box is placed on the table and the unaffected hand is placed in front of the mirror while the affected hand is placed on the other side of the mirror. The distance between the hand and mirror should be the same. The patient is instructed to look at the mirror and concentrate on the exercise. In the first 5 minutes the patient was oriented to watch the reflection of the unaffected hand in the mirror while doing exercise and next 10 minutes, the patient was instructed to do the movement on the hand and still concentrate on the mirror. Certain tasks are done with Pebbles, coin, smiley ball, and pegboard.

\section{Statistical analysis}

Paired t-test was used to analyze significant changes within the group and Unpaired t-test to analyze significant changes between groups.

\section{Outcome measures}

The outcome is assessed by Fugl-Meyer assessment scale.

\section{RESULTS}

The results showed that mean value of TMBT (pretest -5.733 , post-test- 9.86) and SD of (pre-test1.67 , post-test- 1.92) and in CIMT (pre-test-6.3, posttest-11.13) and SD (Pre-test-1.45, Post-test -1.36).
Table 1: Comparing the mean value of constraintinduced movement therapy group by fugl-Meyer scale pre and post-treatment

\begin{tabular}{|c|c|c|c|}
\hline CIMT & Mean & SD & P-value \\
\hline Pre & 6.3 & 1.45 & \multirow{2}{*}{$<0.0001$} \\
\hline Post & 11.13 & 1.36 & \\
\hline
\end{tabular}

Table 2: compare the mean value of task-based mirror therapy of full Meyer scale pre and posttreatment

\begin{tabular}{|c|c|c|c|}
\hline TBMT & Mean & SD & P-value \\
\hline Pre & 5.733 & 1.67 & \multirow{2}{*}{$<0.0001$} \\
\hline Post & 9.86 & 1.92 & \\
\hline
\end{tabular}

\section{DISCUSSION}

This study aimed to provide an overview of mirror therapy and constraint-induced movement therapy for hemiplegic upper limb function. This study could give a sufficient level of evidence in hemiplegic stroke patients for upper limb function. The present literature review shows that there are many ways to modify, the treatment protocol for upper limb hemiplegic stroke (10). During detailed study selection some articles were excluded, as few studies were not focused on a specific outcome and modified CIMT were also excluded. As only a small number of studies were available on constraint-induced movement therapy while searching when compared to mirror therapy (11). CIMT is mostly used in hemiplegic cerebral palsy and not in hemiplegic stroke. And hence further research showed is done which focuses on the constraint-induced movement therapy in hemiplegic stroke.

There was a limited article for CIMT, further research should focus on constraint-induced movement therapy. Our results show more results on mirror therapy, but constraint-induced movement therapy contains only a few results and hence further research should be done. So, we conclude by saying that mirror therapy shows many improvements in hemiplegic upper limb function when compared to constraint-induced movement therapy. Mirror therapy is a treatment method that relies on a patient's observation of their movements to visually trigger the functional recovery of an affected limb (12). Although mirror therapy is effective at improving upper extremity functions, relative to control patients. The improvements in upper limb function observed here similar to those observed in other studies (13). The studies reported a 33\% improvement in upper extremity function, based on FMA in two chronic patients receiving combined treatment involving imagination and wrist and finger flexion and extension (14).

Stroke is one of the major causes of the disability and impairment of muscle strength after a stroke therapeutic challenge for the patient guardians and 
specialists in rehabilitation therapy. In particular, the learned nonuse of the affected upper limb is characterized by the ability to use the less affected upper limb to habitually perform the functional task (15-17). If hemiplegic patients use the unaffected upper limb, they will lose functional independence. This leads to the speculation that the patient would increasingly use the hemiplegic upper extremity and eventually would achieve functional recovery if they concomitantly receive short terms intensive rehabilitation treatment such as constraint-induced movement therapy and task-based mirror therapy following the onset of symptoms. This intensive rehabilitation treatment for the upper extremity hand training may be based on structural plasticity.

In this study, the patients with hemiplegic stroke with upper extremity involvement were included. Further, they divided into two groups one group was given task-based mirror therapy and another group was given constrained induced movement therapy. The treatment was given for 4 weeks and after that, the patient with TBMT showed $(9.86 \pm 1.92)$ and with CIMT showed $(11.13 \pm 1.36)$. Both groups showed good improvement, but more improvement showed in the CIMT group when compare to the TBMT group. Based on this result, the CIMT group showed a significant improvement in hand function and to perform activities of daily living compared to the TBMT group. The hand function training in the CIMT group was repetitive, structural practice intensive therapy in the more affected arm, restraint if the less affected. This finding is attributed to the recovery of the hand function. Improvement in selfcare is one of the most important aspects of performing the activity of daily living. The American Stroke Association has returned that Taub's therapy is at the forefront of a revolution in what is regarded possible in terms of recovery. One study showed that using transcranial magnetic stimulation that the excitable cortex of the affected cortex in adult patients doubled in size after 12 days of therapy. As a result of the patient engaging in repetitive exercises with the affected limb, the brain grows new neural pathways.

\section{CONCLUSION}

The result showed that mean value of TMBT (pretest-5.733, post-test-9.86) and SD (pre-test-1.67, post-test-1.92) and in CIMT mean (pre-test-6.3, posttest-11.13) and SD (pre-test-1.45, post-test-1.36). The study shows that patients with the CIMT group showed more improvement than the TBMT group.

\section{CONFLICT OF INTEREST}

No conflict of interest was declared by authors.

\section{REFERENCES}

1. Thieme, H., Bayn, M., Wurg, M., Zange, C., Pohl, M., Behrens, J. Mirror Therapy for patients with severe arm paresis after stroke- a randomized controlled trial. Clinical Rehabilitation 2013; 27(4):314-324.

2. Pang, M. Y., Haris, J. E., Eng, J. J., A Community based upper extremity group exercise program improves motor function and performance of functional activities in chronic stroke: a randomized controlled trial. Arch Phys Med Rehabil. 2006; 87:1-9.

3. Parker, V. M., Wade, D. T., Hewer, R. L., Loss of arm function after stroke: Measurement, frequency, and recovery. Int Rehabil Med. 1986; 8: 69-73.

4. Olsen, T. S. Arm and leg paresis as outcome predictors in stroke rehabilitation. Stroke. 1990; 21: 247-251.

5. Altschuler, E. L., Wisdom, S. B., Stone, L., Foster, C., Galasko, D., Llewellyn, D. M., et al., Rehabilitation of hemiparesis after stroke with a mirror. Lancet. 1999; 353: 2035-2036.

6. Rizzolatti, G., Fabbri-Destro, M., Cattaneo, L. Mirror neurons and their clinical relevance. Nature clinical practice neurology. 2009; 5(1): 24-34.

7. Reiss, A. P., Wolf, S. L., Hammel, E. A., McLeod, E. L., Williams, E. A. Constraint-induced movement therapy (CIMT): current perspectives and future directions. Stroke research and treatment. 2012 Apr 17; 2012.

8. Sutbeyaz, S., Yavuzer, G., Sezer, N. Mirror therapy enhances lower-extremity motor recovery and motor functioning after stroke: a randomized control trial. Archives of physical medicine and rehabilitation. 2007; 85: 555-559.

9. Yavuzer, G., Selles, R., Sezer, N. Mirror therapy improves hand function in subacute stroke: a randomized controlled trial. Archives of physical medicine and rehabilitation. 2008; 89: 393-398.

10. Stevens, J. A., Using motor imagery in the rehabilitation of hemiparesis. Archives of physical medicine and rehabilitation. 2003; 84: 1090-1092.

11. Taub, E., Miller N. E., Novack T. A., Cook E. W., Fleming W. C., Nepomuceno C. S., et al., Technique to improve chronic motor deficit after stroke, Arch Phys Med Rehabil 1993; 7: 347- 354. PMIT: 8466415.

12. Pomeroy, V., Aglioti, S. M., Mark, V. W., McFarland, D., Stinear, C., Wolf S. L., et al., Neurological principles and rehabilitation of action disorders rehabilitation interventions. Neurorehabilitation and Neural Repair. 2011; 25(5 suppl): 33S-43S.

13. Ramachandran, V. S., Ramachandran, D., Synaesthesia in phantom limbs induced with mirrors. Proceedings of the Royal Society of London B: Biological Sciences. 1996; 263(1369): 377-386.

14. Brodie E. E., Whyte, A., Niven C. A., Analgesia through the looking-glass. A randomized controlled trial investigating the effect of viewing a ,virtuale limb upon phantom limb pain, sensation, and movement. European Journal of Pain. 2007; 11(4): 428-436.

15. Diers, M., Christmann, C., Koeppe, C., Ruf, M., Flor, H. Mirrored, imagined, and executed movements differentially activate sensorimotor cortex in amputees with and without phantom limb pain. Pain. 2010; 149(2): 296-304.

16. Byl, N. N., McKenzie, A. Treatment effectiveness for patients with a history of repetitive hand use and focal hand dystonia: a planned, prospective follow-up study. Journal of Hand Therapy. 2000; 13(4): 289-301.

17. Smorenburg, A. R., Ledebt, A., Deconinck, F. J., Savelsbergh, G. J. Matching accuracy in hemiparetic cerebral palsy during unimanual and bimanual movements with (mirror) visual feedback. Research in Developmental Disabilities. 2012; 33(6): 2088-2098.

18. Ramachandran, V. S., Seckel, E. L., Using mirror visual feedback and virtual reality to treat fibromyalgia. Medical Hypotheses. 2010; 75(6): 495-496.

19. Jack, D., Boian, R., Merians, A. S., Tremaine, M., Burdea, G. C., Adamovich, S. V., et al., Virtual reality-enhanced stroke rehabilitation. IEEE transactions on neural systems and rehabilitation engineering. 2001 Sep; 9(3): 308-318. 
20. Franck Johan, A., Timmermans Annick, A. A., Seelen Henk, A. M. Effects of a dynamic hand orthosis for functional use of the impaired upper limb in sub-acute stroke patients: A multiple single cases experimental design study. Technology and Disability. 2013; 25(3): 177-187. 